\title{
Représentations des enseignants sur l'intégration de la langue de référence en classe de FLE
}

\section{Representations of Teachers on the Integration of the Reference Language in the FFL Class}

\author{
Youcef BACHA ${ }^{1}$ (D), Hakim MENGUELLAT² ${ }^{(0)}$
}

'Doctorant, Université de Ali Lounici - Blida 2, Faculté des Lettres et des Langues, Département de Français, Blida-Algérie ${ }^{2}$ Maître de conférences adjoint, Université de Ali Lounici - Blida 2, Faculté des Lettres et des Langues, Département de Français, Blida-Algérie

ORCID: Y.B. 0000-0002-9312-6504; H.M. 0000-0003-2007-4296

\section{Corresponding author: \\ Youcef BACHA, \\ Université de Ali Lounici - Blida 2 Faculté des Lettres et des Langues, Département de Français, Blida-Algérie E-mail: bachayoucef2016@gmail.com}

Submitted: 14.02 .2020

Accepted: 17.04 .2020

Citation: Bacha, Y. \& Menguellat, H. (2020). Représentations des enseignants sur l'intégration de la langue de référence en classe de FLE. Litera, 30(1), 199-221. https://doi.org/10.26650/LITERA2020-0031

\begin{abstract}
RÉSUMÉ
Notre article s'inscrit dans la perspective de la sociodidactique et la didactique du plurilinguisme qui promeut la pluralité linguistique et culturelle dans le contexte éducatif. Que l'on soit monolingue ou plurilingue, les représentations nous envahissent. Qu'on veuille ou non, elles manipulent nos perceptions et nous guident dans la façon de nommer et d'interpréter les différents aspects de notre réalité. Dans ce contexte, les représentations sur les langues, y compris les langues premières influencent en fait l'enseignement-apprentissage en classe. En effet, elles sont considérées comme un système de perception ou des images mentales construites par les locuteurs sur les langues acquises ou apprises. C'est pourquoi, la prise en compte des représentations des apprenants et des enseignants est un facteur important pour faciliter l'apprentissage et la transmission des connaissances. Dans cette présente contribution, nous mettons l'accent sur les représentations que se font les enseignants quant à l'intégration de la langue de référence en classe de FLE. Nous sommes partis de cette question : quelles représentations se font les enseignants de la langue de référence en classe de FLE ? Pour répondre à cette question, nous émettons l'hypothèse suivante : les enseignants auraient souvent des représentations négatives envers la langue de référence. Pour ce faire, nous avons opté pour un entretien semi-directif. Au terme de notre étude, nous avons abouti aux résultats montrant qu'il y a un « hiatus » entre le discours sur les pratiques de classe et les pratiques effectives en classe.
\end{abstract}

Mots-clés: Représentations, langue de référence, apprentissage du FLE, continuum linguistique, didactique du plurilinguisme

\section{ABSTRACT}

The present article falls within the perspective of sociodidactics and the didactics of plurilingualism which promotes the linguistic and cultural plurality in the context of education. Whether we are monolingual or multilingual, representations strike us. Whether we want it or not, they manipulate our perceptions and guide us in how to name and interpret the different aspects of our reality. In this context, the representations of languages, including the first languages, actually influence teaching-learning in the classroom. Indeed, they are considered as a system of perception or mental images that are constructed by speakers on the languages 
acquired or learned. Therefore, taking into account the representations of learners and teachers is an important factor to facilitate the teaching-learning process and the transmission of knowledge. In this present contribution, we mainly focus on the representations made by teachers regarding the integration of the reference language in the French as a foreign language class. Hence, we started from this question: what representations do teachers make of the reference language in the French as a foreign language class? To answer this question, we hypothesize that teachers would often have negative representations towards the integration of the reference language in the teaching-learning process. To do this, we opted for a semi-structured interview. At the end of our study; we came to the conclusion that there is a "gap" between the discourse on class practices and actual practices in the classroom.

Keywords: Representations, reference language, learning FFL, linguistic continuum, didactics of plurilingualism

\section{EXTENDED ABSTRACT}

Our article falls within the perspective of sociodidactics and the didactics of plurilingualism which promotes the linguistic and cultural plurality in the context of education.

Whether we are monolingual or multilingual, representations strike us. Whether we want it or not, they manipulate our perceptions and guide us in how to name and interpret the different aspects of our reality.

In this context, the representations of languages, including the first languages, actually influence teaching-learning in the classroom. Indeed, these representations are considered as a system of perception or mental images which are constructed by speakers on the languages acquired or learned. Therefore, taking into account the representations of learners and teachers is an important factor to facilitate the learning process and the transmission of knowledge between interlocutors.

In the present article, we would like to focus on the teachers' representations of the integration of the reference language in FFL learning. In this perspective, we set out to describe the Algerian context which is practically marked by a plurality of languages and cultures: classical or school Arabic, dialectal Arabic or Darija, Tamazight with its different varieties, French, etc. This sociolinguistic situation is clearly marked by a phase shift between official language policy or the imposition of state monolingualism and co-present linguistic diversity or de facto plurilingualism. This linguistic complexity and diversity ipso facto leads to divergent representations among speakers, in this case, teachers of the third year of the secondary school. These representations could be negative or positive.

In this context, we found that teachers always base their class practices on received ideas or stereotypical representations, in particular with regard to the integration of the reference language in the learning of the French language. 
To investigate the representations of teachers, we started with the following question: what representations do teachers make of the integration of the reference language in the French as a foreign language class?

To answer this question, we only postulate that teachers of the third year in the secondary school have «negative» behaviors towards the use of the reference language in the French as a foreign language class.

To conduct this study, we opt for a semi-structured interview, a data collection tool, in order to highlight the different representations that teachers make of the integration of the reference language in the learning of FFL.

We carried out a qualitative method to respectively analyze the data obtained according to two main axes divided into several criteria: the representations on the reference language and the impact of this language on the learning of FFL.

Our findings show that the reference language, in fact, has many functions; we cite among others, the function of exclusion and self-exclusion, the fun function, the socio-pragmatic function and the identity function. However, these different representations run counter to their actual practices in the classroom, namely the impact of the reference language on FFL language learning. Teachers declare that the reference language plays a pedagogical-didactic role because it simplifies learning and helps understanding: «the didactization» of learning FFL.

In addition, the teachers' speeches are marked by the inclusion of certain terms related to the reference language. In fact, teachers use the Arabic language from time to time to explain certain words or to translate their ideas. To this end, we have set a "continuum» which lists the teachers according to the frequency of the reference language's use in their verbal declarations in the classroom.

From this analysis, we can deduce that the teachers' representations go against their real practices (discourse) in the classroom.

Hence, monolingualism is only a representation made by the teacher in the FFL class, while plurilingualism is the real fact. 


\section{Introduction}

Nous tenons à montrer du doigt, dans le présent article, les représentations que se font les enseignants de l'intégration de la langue de référence (désormais LR) dans I'apprentissage du FLE. En effet, les enseignants fondent toujours leurs pratiques sur des idées reçues, celles-ci pourraient être le fondement de toute pratique pédagogique en classe. Pour interroger les représentations des enseignants, nous sommes partis du questionnement suivant: quelles représentations se font les enseignants de l'intégration de LR en classe de FLE. Pour répondre à cette question, nous postulons que les enseignants de troisième année secondaire (dorénavant $3 \mathrm{AS}$ ) auraient des comportements désapprobateurs envers I'usage de LR en classe de FLE.

Pour ce faire, nous optons pour un entretien semi-directif afin de mettre en relief les différentes représentations que se font les enseignants.

Dans cette perspective, nous nous attelons à définir certains concepts théoriques pour mettre en lumière la diversité linguistique qui caractérise le contexte algérien et les différentes représentations que se font les acteurs sur les langues co-présentes en l'occurrence LR.

\section{Complexité du contexte sociolinguistique en Algérie}

Le paysage sociolinguistique algérien s'est façonné, au fil du temps, par maintes civilisations qui sont dérivées des belligérances successives : phénicienne, carthaginoise, turque, espagnole, française... A ce titre, cette cohorte civilisationnelle engendre ipso facto un mélange de nombreuses variétés linguistiques.

De facto, ce pays est (re)devenu, à l'ère actuelle, un champ-carrefour qui décèle fondamentalement un plurilinguisme et un pluriculturalisme incontestés, comme en témoignent Ph. Blanchet et P. Martinez :

L'Algérie est un espace dans lequel ont cours des variétés linguistiques multiples : arabe algérien avec différentes variantes locales, l'arabe dit «classique » qui est la langue de l'école, le français, le berbère avec différentes variantes locales (tamazight, kabyle, chaoui, targui), expression d'une diversité culturelle constitutive. (2010, p. 205) 
Dans cette lignée, la question du plurilinguisme constitue incontestablement un débat privilégié, du fait que tout locuteur algérien se dote de nombreuses langues/ variétés langagières. Cette situation d'enchevêtrement et de croisement des langues, se faisant jour, en Algérie, a une incidence incontournable sur les pratiques langagières en classe.

L'apprenant algérien est, depuis sa tendre enfance, confronté à une situation structurellement plurilinguistique (Sebaa, 2015, p. 70) : I'arabe dialectal et le Tamazight. Puis au cours de la scolarisation, il s'approprie d'autres langues normées telles que l'arabe littéraire ou classique, le français et les autres langues étrangères. Cette «mosaïque linguistique» dote l'apprenant d'un bi-/plurilinguisme qui le baigne dans un contexte marqué par la diversité et la complexité langagière.

Effectivement, l'acquisition des langues et/ou varitétés sociales telles que l'arabe dialectal et le tamazight dans le contexte berbérophone et l'apprentissage des langues étrangères à l'école déconstruit le mythe babylonien patent ledit «monolinguisme» au profit d'un plurilinguisme latent.

Du reste, I'apprentissage de nouvelles langues, en l'occurrence le français langue étrangère entraîne un effet d'engouement-étonnement chez l'apprenant, en d'autres termes l'apprenant reçoit un nouveau système linguistique en partie ou entièrement différent de celui de sa propre langue; ce fait le convie à faire écho à la langue source comme bouée de sauvetage ou langue d'appoint en vue de s'accommoder au déséquilibre, de s'acclimater aux effets fluctués et de s'enfuir du « choc linguistique » que pourraient entraîner l'apprentissage des langues étrangères. A cet effet, l'apprenant produit inéluctablement un « syncrétisme » langagier ou un métissage de codes.

\section{Représentations sociales}

Compte tenu du caractère transversal et multipolaire du concept de représentations, il s'est inscrit dans de nombreux champs scientifiques. En effet, nous reconnaissons d'abord sa genèse dans les travaux d'E. Durkheim pour désigner une représentation idéale présente chez un collectif. «Durkheim(1895) fut le premier à identifier de tel objet, comme productions mentales sociales relevant d'une étude de l'« idéalisation collective » (Jodelet, 1989, p. 35). 
Ayant vu le jour dans le domaine de la sociologie, les représentations sociales désignent des images mentales forgées par un individu ou un groupe portant principalement sur des objets ou des sujets sociétaux. « les représentations expriment ceux (individus ou groupes) qui forgent et donnent de l'objet qu'elles représentent une définition spécifique » (lbid., 1989, p. 35).

Elles sont considérées comme système de gestion de notre rapport au monde et normes régissant nos interprétations des faits sociétaux. Effectivement, elles façonnent notre regard en canalisant les préalables représentationnels et en subordonnant toute position interprétative prise par les locuteurs envers les objets du monde, D. Jodelet (1989) le note : "On reconnaît généralement que les représentations sociales, en tant que système d'interprétation régissent notre relation au monde et aux autres, orientent et organisent les conduites sociales » (p. 36).

Elles ne changent pas uniquement nos regards portés sur le monde, mais surtout elles forgent nos identités en exerçant un pouvoir sur l'assimilation des connaissances, sur leur diffusion et sur notre rapport à l'Autre.

D'autant plus que les représentations sont génériques et généralisatrices d'actions, dans la mesure où l'individu est imprégné des idées reçues qui régissent ses actions au quotidien. "La représentation détermine à la fois le stimulus et la réponse, qu'il n'y a pas de coupure entre l'univers extérieur et l'univers intérieur de l'individu » (Jodelet, 1989, p. 39).

Ce concept est progressivement introduit dans le domaine de la sociolinguistique et qui occupe, par conséquent, une place de choix en problématisant de nombreux travaux de recherche portant sur les langues-cultures et leur enseignement-apprentissage. De ce fait, les représentations sociolinguistiques semblent être un cumul d'images caricaturales projetées sur les langues et leur catégorisation, $\mathrm{H}$. Boyer entérine cette idée : "Les représentations sociolinguistiques sont pour nous une catégorie de représentations sociales collectives, donc partagées » (2001, p. 41).

Dans cette perspective, nombreux sont les travaux qui traitent du fonctionnement des langues, leurs relations, leurs statuts, ainsi que la posture qu'adopte l'individu à l'égard ou à l'encontre de telle langue. V. Castellotti et D. Moore expliquent que « Les sociolinguistes, en particulier, ont mené de nombreux travaux sur les attitudes et les représentations des sujets vis-à-vis des langues, de leur nature, de leur statut ou de leurs usages» $(2002$, p. 09). 
Par ailleurs, la minorisation et/ou la minoration des langues (Rispail, 2017, p. 88) et les frontières qui les séparent ne sont que des représentations portées par les locuteurs, et celles-ci, au vu de leur caractère leitmotiv, légitiment ou stigmatisent par conséquent telle langue au profit ou au détriment d'une autre en modifiant le contexte sociolinguistique qui, à son tour, façonne les comportements des locuteurs. Le cas de l'arabe standard dit «classique» qui est réputée en tant que langue officielle sans être la langue mise au quotidien par les locuteurs ou les langues premières -l'arabe dialectal appelé daridja, maghribi (Elimam, 2015), etc. et le tamazight- qui sont minorisées et perçues impures. A cet égard, P. Bourdieu estime que : « la langue, le dialecte ou l'accent sont l'objet de représentations mentales, c'est-à-dire acte de perception et d'appréciation, de connaissance ou de reconnaissance, où les agents investissent leurs intérêts et leurs présupposés [...] » (1982, p. 135).

\section{Objectif et méthodologie}

Nous avons opté pour les enseignants de troisième année secondaire de langue française car ils sont, par leur long parcours et leur conscience linguistique, davantage représentatifs de la richesse du répertoire plurilingue : arabe littéraire, dialectal, français, anglais... Ces acteurs nous renseignent sur leurs représentations et leurs pratiques en classe.

Cette pluralité langagière articulée sur le champ de la didactique permet de faire émerger les représentations des enseignants sur LR et son impact sur l'apprentissage du FLE.

Rappelons que notre échantillon est constitué de 5 enquêtés. Les 5 enseignants travaillent dans des établissements distants et différents. Pour une représentativité de notre échantillon, nous avons opté pour des enseignants coordinateurs assurant une formation interne aux enseignants stagiaires.

Notre analyse s'articule autour de deux axes principaux :

$>$ les représentations sur les langues de famille;

> l'impact de LR sur l'enseignement-apprentissage du FLE.

Nous transcrivons d'abord intégralement les discours des enquêtés, puis nous les décrirons afin d'en tirer davantage profit plutôt que de les analyser. 
Nous clôturerons l'interprétation des entretiens par une analyse globale et récapitulative.

\section{Constatation}

\section{Représentations sur l'intégration des langues de famille}

Il s'agit de voir les tendances représentationnelles que portent les enquêtés sur I'intégration des langues de famille en classe de FLE et les raisons qui les incitent à adopter telle ou telle attitude envers ces langues.

\subsection{Attitudes envers la langue de référence en classe de FLE}

Le premier enquêté adopte une attitude ambivalente envers les langues de famille en classe : elles constituent pour lui une arme à double tranchant, en d'autres termes leur usage est avantageux quand elles sont moins usitées en classe que la langue d'enseignement en l'occurrence le FLE.

-Parfois je trouve que c'est/gênant parfois puisque mes élèves parlent beaucoup plus l'arabe que le français ça va donner une influence négative sur leur apprentissage/ et parfois je trouve ça bénéfique alors comment dirai-je/c'est une arme à double tranchant/c'est une arme à double tranchant// porte des bienfaits parfois nocif

Il adopte, d'une part, une attitude euphorique envers cette langue lorsqu'il s'agit d'une situation-problème, car c'est le seul moyen auquel il se réfère dans des cas d'« aporie».

-parfois c'est c'est bénéfique pac'que/ dans les cas de blocage on doit parler en arabe pour débloquer/pour euh briser cette bloc de roche/d'accord! (rire)/ pour que le message passe

Toutefois, il a, d'autre part, une visée négative envers cette langue lorsque son usage devient abusif et récurrent en classe.

-Parfois يغي (c'est-à-dire) n'est pas bénéfique pac'que/ces apprenants vont/ j sais pas comment dire ça mais/ils vont utiliser beaucoup plus l'arabe que le français et notre objectif c'est apprendre le français vous voyez! 
L'enquêté s'aperçoit que l'usage unique de la langue française en classe peut provoquer une vision d'étonnement voire de «choc linguistique», dans le sens où le discours de l'enseignant est en entier en déphasage avec la compétence de l'apprenant. Et pour renouer ce lien rompu, l'enseignant n'a qu'à parler la langue arabe, quel soit littéral ou dialectal.

-Bénéfique parc'que parfois on est// bloqué d'accord! on est bloqué on peut rien faire/ alors les élèves ne comprennent pas comme s'il ya quelqu'un qui parle par exemple/si par exemple nous sommes des francophones ou parfois si comme quelqu'un qui parle le chinois/alors le chinois c'est la langue la plus difficile dans le monde/vous comprenez? Alors parfois je trouve que/ils sont en train de voir quelqu'un qui vient d'une autre planète voilà !/on est bloqué/ dans ce blocage parfois j'interviens en arabe pour débloquer ce blocage

L'attitude positive que porte l'enseignant envers la langue arabe, en classe, est associée davantage à une exigence didactique plutôt qu'un choix linguistique délibéré.

-Parfois je favorise/je suis tout à fait d'accord pour l'utilisation de l'arabe dans l'apprentissage du FLE en Algérie/ c'est ça ! on a pas du choix

La deuxième enquêtée a une représentation positive vis-à-vis de l'intégration de cette langue en classe, car c'est la langue la plus utilisée par les apprenants au quotidien.

-Je sais pas mais je pense que c'est normal/je pense que c'est normal parce que entre eux ils ne peuvent pas se se/ منيش عارفا (je ne sais pas)/ discuter en français impossible

Aussi, l'usage de l'arabe en classe est relié au contexte géolinguistique et au niveau instructif de l'apprenant « niveau bas».

-Normal normal/ même lorsque parfois je parle/ premièrement l'élève son niveau il est vraiment bas donc tu dois/ je peux pas crier sur un élève parce qu'il a parlé en arabe/ c'est la langue maternelle et en plus c'est la langue/ donc cette situation et dans cette région c'est pas comme les autres régions/ donc ici je peux pas l'obliger à se taire et de ne pas parler (rire)// 
L'enseignante voit, d'une part, que l'usage de la langue arabe a droit de cité en classe car elle constitue, pour l'apprenant, un outil libérateur de sa parole ; et d'autre part, l'enseignant doit se priver de telle ou telle position contraignant le discours de l'apprenant compte tenu de son niveau «déplorable » en FLE.

-je pense que c'est normal c'est parce que la langue française pour les élèves/ c'est pas le problème de l'arabe/ problème c'est de la langue française/ عند (a) pour lui il a le droit de parler en arabe/ et pour lui il n'a pas une autre façon de s'exprimer à part l'arabe/ أl (je) je pense c'est normal/

-j'ai pas le droit de les empêcher/sont libres je pense ils sont libres/surtout que leur niveau ne leur permet pas de discuter en français avec leurs camarades/

L'enquêtée $n^{\circ} \mathbf{3}$ s'aperçoit que la présence de la langue arabe en classe est nécessaire pour expliquer une situation difficile ou surmonter un obstacle.

Li- (je) pour moi l'utilisation de la langue maternelle en classe/ c'est c'est pour moi je pense c'est important/ pourquoi ? pour moi c'est important on parle pas toute la séance avec la langue maternelle/mais on essaie d'expliquer les mots difficiles avec la langue maternelle/ pour moi c'est important/ -La vérité normal

En outre, l'enseignante adopte une attitude favorable à l'égard de la présence de LR en classe.

-Oui ! ouaih ! la vérité ça c'est/c'est vrai ouaih!

L'enquêtée $\mathrm{n}^{\circ} \mathbf{4}$ exprime une attitude négative vis-à-vis de l'intégration des langues de famille en classe, car leur usage est « inutile». Pour elle, la langue à apprendre doit s'expliciter par elle-même, autrement dit la fonction structuraliste et métalinguistique émane de la langue enseignée en question sans pour autant se référer à d'autres langues, en l'occurrence le tamazight et l'arabe.

-C'est inutile/ par exemple dans un cours de français d'utiliser le tamazight ou l'arabe c'est inutile/complètement inutile c'est la même chose avec l'arabe/ c'est la même chose avec l'arabe/ moi je vois qu'il faut apprendre une langue par elle-même/ c't'à dire la même langue pas par une autre langue/ je sais pas moi/ c'est inutile/ inutile complètement inutile je sais pas/ pac'que 
l'apprenant n'apprend rien à travers ces habitudes/ non il est inutile pour l'apprenant/ utiliser amazight pour parler français ou bien arabe pour apprendre tout français/il n'apprend rien comme ça (rire) -Le français s'apprend par le français par le français

Aussi, la visée pragmatique de la communication empêche l'enseignante de faire écho à LR, car toute situation de communication extrascolaire, déclare-t-elle, à laquelle sera confrontée l'apprenant se mènera en français. C'est pour cette raison que le rôle de $L R$, à ses yeux, se voit minimisé voire dévalorisé.

-Je pense qu'il n'est pas possible d'apprendre une langue par une autre/ pourquoi ? Pac'que quand il rencontre une/ par exemple se retrouve dans une situation ou de communication/par exemple il apprend là/dans une situation de communication/و/ou) il doit répondre en français il subit des questions/ je sais pas moi sur des communications des contacts avec des étrangers/il fait réflexion/il fait penser toujours aux mots arabes qu'il a utilisés en classe/donc c'est inutile/ par exemple je sais pas moi/son ami qui est en contact avec lui/ ça français par exemple/ parce qu'on prépare l'apprenant à des situations de communication/alors il fait une communication avec un français ou je sais pas moi ou un étranger est-ce/est-ce que il peut utiliser l'amazight ou l'arabe en correspondant avec cette personne?/est-ce que c'est utile/il est inutile/il est complètement inutile donc je trouve que c'est pas possible pac'que notre objectifc'est le préparer pour des situations de communication afin de trouver un apprenant authentique/ ou je ne sais pas ou autonome si vous voulez!

\subsubsection{Fonction identitaire}

L'enquêté $n^{\circ} \mathbf{1}$ considère que l'arabe dialectal est la langue de la poésie et de la littérature, en revanche il accorde un immense privilège à l'arabe littéraire ou « classique ». L'usage de l'arabe dialectal, dit-il, exerce une fonction explicative du discours pédagogique car il est plus proche de la culture et l'environnement de l'élève.

-Si si personnellement/j'aime Abdrrahmane El Majdoub/alors c'est un grand poète de la poésie populaire d'accord! mais personnellement/j'aime la poésie arabe académique/ littéraire/ classique comme dirai-je El Motanabi Chafiî etc. (E.1) 
-je parle l'arabe dialectal pour qu'ils me comprennent par exemple/quand je dis par exemple/parle du dérivéde/je sais pas/de lait par exemple ses dérivés du beurre نقولو دهان نقولو زبل directement// d'accord ! c'est plus proche à leur environnement culturel on peut dire/ voilà (E.1)

-L'arabe quand même l'arabe représente notre identité (E.1)

Quant à l'enquêtée ${ }^{\circ} \mathbf{4}$ voit que l'utilisation de l'arabe en classe est liée essentiellement à une dimension identitaire de l'apprenant.

-parfois l'arabe/ puisque on est des arabes/ les apprenants comprennent mieux (E.4)

L'enquêté $n^{\circ} 5$ pose une interrogation affirmative sur l'identité des apprenants et déclare à ce propos que le recours à l'arabe dialectal en classe s'explique par le fait de son utilisation massive au foyer familial.

-Pour faire référence/ qui sommes-nous ? nous sommes des Algériens nous utilisons dans nos maisons ce dialecte (E.5)

\subsubsection{Fonction métalinguistique}

La mise en œuvre des langues de famille en classe a pour objectif d'expliciter ou notamment de «didactiser» la langue à apprendre, car elles sont vues comme une "source de savoir », dont témoignent les enquêtés 1, 2, 3 et 5 .

-Alors parfois je trouve que/ils sont en train de voir quelqu'un qui vient d'une autre planète/ voilà ! on est bloqué dans ce blocage parfois j'interviens en arabe pour débloquer ce blocage (E. 1)

-Je trouve parfois elle facilite l'apprentissage/ elle le facilite/veut dire ça aide à apprendre une langue étrangère/ puisque ça reste toujours une langue étrangère/ ce n'est pas une langue maternelle/ c'est pour cela qu'on doit l'expliquer/ parfois par des mots traduits dans la langue maternelle de l'apprenant/on a pas le choix parfois (E.1)

-Je demande à tout le monde de me dire comment on dit ce qu'il a dit leur camarade en arabe dialectal/ de me le traduire en français/comme ça au moins ils vont comprendre/ puisque la traduction/ pour moi la traduction c'est une source de savoir (E.1) 
-alors pour moic'est un source de savoir/puisque ça facilite la compréhension/ ça/ça donne/ je sais pas comment dirai-je ça? // moi j'ai pas trouvé d' mots/ c'est un avantage/ pour l'apprenant c'est un avantage (E.1)

-L'explication V (non) j'essaie de le faire expliquer en français/بصح (mais) نشوف فيه بلبي راه شوي (si je vois qu'il n'arrive pas à me comprendre) je l'explique en arabe (E.2) -lorsque même l'élève qui est compétent n'arrive pas à donner le mot en arabe/ n'arrive pas à comprendre le sens/donc je lance le mot en arabe voilà ! (E.2) - quand je vais expliquer des mots difficiles/j'utilise l'arabe (E.3)

- Lil (moi) pour moi l'utilisation de la langue maternelle en classe c'est/ c'est pour moi je pense c'est important/pourquoi ? pour moi c'est important/ on parle pas toute la séance avec la langue maternelle/mais on essaie d'expliquer les mots difficiles avec la langue maternelle/ pour moi c'est important (E.3) -ll n'a pas compris bien ! on est arrivé à la situation de blocage/ de débloque (rire) je débrouille en arabe! (E.5)

-Pour moi/ le rôle des langues maternelles a un rôle important / pourquoi ?/ c'est un rôle important/pourquoi ? c'est essayer de simplifier le sens les mots difficiles/en général j'explique pas la leçon avec la langue maternelle/j'explique j'ai dit des mots (E.3)

-si je vais l'utiliser pour que je sorte de l'ambiguïté/ pour que je minimise le temps/ pa[R]c'que le mot étudié qu'on veut comprendre prendra beaucoup de temps/toute une situation pour l'expliquer/là peut-être là je vais le dire puisque/l'accès au sens se ferait plus rapidement en dialectal que l'explication en langue française/ beaucoup de temps/ pa[R]c'que comme vous savez la compréhension du mot est très sensible/ très sensible (E.5)

-le dialecte peut l'utiliser comme moyen d'explication du contexte/ ou bien ou du du vocabulaire des mots (E.5)

\subsubsection{Complexité langagière}

L'arabe dialectal est perçu par l'enquêté $\mathrm{n}^{\circ} \mathbf{5}$ comme un mélange de plusieurs langues ou plutôt comme une sorte "d'interférence » de nombreux systèmes linguistiques (lingua franca). Et considère également que cette langue au lieu d'être un vecteur d'apprentissage d'autres langues, elle entrave, en revanche, l'apprentissage de la langue française. Nous citons quelques séquences verbales qui montrent la perception « négative » de l'enquêté. 
-Pourquoi ? pa[R]c'que/Pourquoi ?/ pa[R]c'que au lieu de/c'est-à-dire au lieu de dialecte c'est un mélange plus/plus plurilinguistique/ c'est un mélange/ euh qui qui provient qui provient de plusieurs langues/ avant et autre langue le français/il faut l'acquérir/ quatre ou cinq langues plus la langue française/ ça devient cinq langues à expliquer/ ce qui est ce qui est difficile ce qui gêne ce qui gêne ce qui gêne la compréhension du français/c't'à dire au lieu d'utiliser six langues sans connaître les règles d'une seule/ il faut utiliser une seule et connaître bien ça langue/ c'est bien le français/ (E.5)

-Pluralité de langues/le dialecte c'est une pluralité de langues/ça vient je sais pas moi du Turc/ de l'espagnol/ des gens utilisent maint'nant ce dialecte et ils ne savent pas d'où provient ?/ D'où provient cette langue ce dialecte ce mot ? ils ne savent pas d'où provient? donc là ! est-ce que on va se contenter d'expliquer le français/ ou bien d'où vient ce mot de dialecte/ pourquoi ce partager entre deux? Pourquoi jeter l'élève dans une ambiguïté ?/ comparatif entre une langue écrite et une langue qu'on peut pas écrire//(E.5) -Bien sûr bien sûr!/ et écoutez moi/lo[R]squej'entends ce dialecte je me perds pa[R]c'que/

\subsubsection{Représentations négatives «absurdité langagière»}

L'enquêté $n^{\circ} \mathbf{5}$ s'aperçoit que les dialectes ont une construction linguistique arbitraire, dans la mesure où ils ne disposent pas de règles et de normes qui les régissent.

Je ne vois pas des règles dans les dialectes/je ne vois pas des règles/ et puis je trouve que ça n'a pas de féminin/ c'est-à-dire aléatoire/ça n'a pas de féminin/ça n'a pas de pluriel/ ça n'a pas de règles

\subsubsection{Fonction socio-pragmatique}

La fonction assignée à $L R$ a une finalité pragmatique : traduire un mot et se mettre au niveau des apprenants, en d'autres termes la mise en œuvre de cette langue est vue comme véritable appui pour l'enseignement du FLE, dans le sens où le cours ne saurait s'effectuer uniquement en langue française, du fait que la centration sur l'apprenant et sur sa/ses compétence(s) langagière(s) en classe constitue la pierre angulaire de toute pratique pédagogique. 
-Alors parce que pour apprendre une langue comme je t'ai dit tout à l'heure on doit la traduire la première des choses le premier acte/ c'est traduire ce mot en arabe (E.1)

-Pac'que le niveau est faible c'est pour ça j'essaie d'utiliser la langue arabe/ c'est ça! (E. 2)

- Li) (moi) pour moi l'utilisation de la langue maternelle en classe/ c'est c'est pour moi je pense c'est important/ pourquoi ? pour moi c'est important on parle pas toute la séance avec la langue maternelle (E.3)

\subsubsection{Fonction de désapprentissage}

Les enquêtés $n^{\circ} \mathbf{4}$ et $n^{\circ} 5$ avancent que l'usage de LR est inutile et qu'elle ne soutient pas l'apprentissage de la langue française. Au contraire, elle entraîne en partie ou en entier un obstacle d'apprentissage surtout lorsqu'il s'agit d'une situation de communication authentique avec un locuteur exolingue.

-C'est inutile/ par exemple dans un cours de français d'utiliser le tamazight ou l'arabe/c'est inutile/complètement inutile c'est la même chose avec l'arabe c'est la même chose avec l'arabe/ moi je vois qu'il faut apprendre une langue par elle-même/ c't'à dire la même langue pas par une autre langue/ je sais pas moi/ c'est inutile/ inutile complètement inutile/ je sais pas pac'que l'apprenant n'apprend rien à travers ces habitudes/non il est inutile pour l'apprenant/ utiliser amazight pour parler français ou bien arabe pour apprendre tout français// il n'apprend rien comme ça (rire) (E.4) -Je ne trouve pas// non non non! aucune importance/ une autre langue vivante comme anglais ça aide (E.4)

-L'apprenant n'apprend rien à travers cette mauvaise habitude (E.4) -est-ce est-ce que il peut utiliser l'amazight ou l'arabe en correspondant avec cette personne est-ce que c'est utile ?/il est inutile il est complètement inutile donc je trouve que c'est pas possible pac'que notre objectif c'est le préparer pour des situations de communication afin de trouver un apprenant authentique ou je ne sais pas ou autonome si vous voulez!

-Non non! pa[R]c'que le dialecte pour moi ça n'aide pas/ ça n'aide pas pourquoi ? pa[R]c'que ne sera pas réutilisé/ne sera pas investi dans son travail que ce soit en arabe ou bien en anglais ou bien en français/donc c'est un dialecte qui reste à part qui reste à part et bien sûr et bien sûr qui ya une autre 
signification même le dialecte n'a pas le même sens/ n'a pas le même sens assez précis demandé dès le début de la compréhension/ dans l'explication du mot/je n'accepte pas (E.5) -je ne vois l'utilité de l'utilisation de ce dialecte (E.5) -je n'accepte pas l'utilisation de cette langue en classe/en classeje ne l'accepte pas/ pa[R]c'que que comme je vous ai dit c'est une ambiguïté (E.5) -c'est c'est je pense que c'est un mélange/ je sais pas c'est quelque chose qui n'est pas organisé/ qui qui c'est une utilisation très dangereuse/dangereuse pour la langue et pour l'apprentissage/ (E.5)

\subsubsection{Fonction d'exclusion et d'auto-exclusion}

L'enquêté $\mathrm{n}^{\circ} \mathbf{5}$ déclare que le recours à $\mathrm{LR}$ dévalorise à la fois la figure enseignante et son statut. Pour lui, le dialecte n'obéit à aucune règle ou norme stricte.

donc je n' peux pas/je peux pas je peux pas donner/le prononcer en classe/ pa[R]c'que je $n^{\prime}$ sais pas// pa[R]c'que je sais pas pa[R]c'que l'élève après un jour va dire c'est le professeur qu'il a dit/pour lui le professeur c'est un guide/ c'est un dictionnaire/donc c'est une source fausse /moi je suis pas sûr de l'explication de ce mot (E.5)

-Ça démunie mon statut quoi/ en tant qu'enseignant (E.5)

\section{Impact de la langue de référence}

Dans cette perspective, nous mettons en lumière le rôle que joue LR en classe de FLE. Cette dernière pourrait remplir de nombreuses fonctions pédagogico-didactiques.

\subsection{Bouée de sauvetage ou médium d'enseignement}

Les enseignants ont recours à LR comme ultime solution : sa mise en œuvre constitue un outil facilitateur de l'enseignement-apprentissage de la langue française notamment sur le plan de la compréhension et de l'explication.

-j'utilise l'arabe quand je suis devant un obstacle ou mes élèves ne comprennent pas/voilà j'utilise l'arabe pour diminuer cet obstacle (E.1) -on est bloqué/dans ce blocage parfoisj'interviens en arabe pour débloquer ce blocage (E.1) 
-c'est c'est bénéfique pac'que/ dans les cas de blocage on doit parler en arabe pour débloquer/ pour euh ! briser cette bloc de roche d'accord! (rire)/ pour que le message passe (E.1)

-alors pour briser cet obstacle et un autre obstacle (rire)// on doit chercher le mot dans l'arabe dialectal/ pas l'arabe littéraire ou académique (E.1)

-Je valorise beaucoup l'utilisation de l'arabe dialectal dans certains cas/dans les cas de blocage surtout/ on est dans une situation de blocage on doit débloquer la situation coûte que coûte / on est obligé de passer à la langue dialectale on a pas le choix (E.1)

-On peut pas l'apprendre d'autres manières mais/ c'est un moyen comment dirai-je de sauvetage de secours qu'on utilise/ parfois pour débloquer dans les situations d'extrême urgence (rire)// (E.1)

-l'arabe qu'on utilise en classe/ça facilite la communication entre le professeur/ l'enseignant et l'app[R]enant/(E.1)

-Je parle l'arabe dialectal pour qu'ils me comprennent (E.1)

L'enseignante demande à l'élève le plus compétent en classe de donner l'équivalence sémantique en arabe, et lorsque ce dernier n'arrive pas à trouver la réponse adéquate, elle intervient pour le dire finalement en arabe.

-lorsque même l'élève qui est compétent n'arrive pas à donner le mot en arabe/ n'arrive pas à comprendre le sens/ donc je lance le mot en arabe voilà ! (E.2) -les élèves que j'en ai là/ avec leur niveau ils te poussent ils te poussent à expliquer/ des fois des fois (E.2)

-Si les élèves n'arrivent pas à le dire en arabe c'est moi qui va le dire/ je suis obligé de l'utiliser en arabe (E.2)

L'enquêtée $\mathrm{n}^{\circ} 3$ tente d'expliciter le mot avec de nombreux outils (image, illustration, dessin...), et si toutes les explications sont vouées à l'échec, elle donne le terme finalement en arabe.

-j'essaie de dessiner l'explication de mot/ce n'est pas le mot je peux pas dessiner le mot soit l'explication/ soit j'essaie de parler en arabe/ l'arabe classique et des fois même j'utilise l'arabe dialectal/le dialecte c'est ça! (E.3)

-La première des choses j'essaie d'expliquer le mot en français/ je simplifie l'explication/j'essaie de donner des mots simples/ c'est ça! pour expliquer le mot (E.3) 
L'enseignante demande tout d'abord à l'élève de traduire le mot en arabe. Et quand ce dernier ne saisit pas le sens du mot, elle le traduit, en fait, en arabe. Aussi, elle ajoute que le recours à l'arabe dialectal peut être un gain de temps d'explication pour poursuivre le cours.

-des fois si je trouve le mot est difficile pour l'élève/ qu'est-ce que je vais faire? je lui expliquais en français/ $j$ vais simplifier le mot/si les élèves n'arrivent pas à comprendre le mot dans ce cas/dans ce cas j'essaie soit je demande à un élève en général/j'ai un ou une élève qui sont meilleurs في (en) l'explication et tout/ dans ce cas je demande à un élève de parler en français plutôt de parler en arabe/ d'expliquer le mot en arabe soit en arabe classique soit en arabe dialectal/c'est tout! sinon si tout le monde me regarde comme ça sans comprendre le mot/dans ce cas je prononce le mot en arabe/ (E.3)

-Des fois j'accepte l'utilisation de l'arabe familial ou dialectal/ ou l'arabe classique dans le cas où le mot est difficile/ mais il faut que passe à d'autres points c'est normal/ c'est pas la peine de perdre de temps d'expliquer le mot/ je demande à un élève allez-y tu donnes à vos camarades l'explication en arabe/ و(et) ça y est ! c'est ça mais il est très important/la vérité d'utilisation de la langue maternelle en classe (E.3)

-je suis avec l'utilisation dans des cas urgentes/cas urgent par exemple dans des cas/ un cas par exemple un cas par exemple d'un mot difficile/ $\mathbf{V}$ (ou) un cas urgent c'est tout/ un cas où je peux pas expliquer le mot (E.3)

-J'essaie de trouver l'explication pour quelques mots difficiles/c'est c'est c'est pour ne pas perdre de temps c'est tout (E.3)

L'enquêtée $n^{\circ} 4$ recourt à la langue arabe, que ce soit dialectal ou littéral, pour débloquer une situation-problème après maintes tentations d'explicitation en langue française.

-parfois l'arabe/puisque on est des arabes les apprenant comprennent mieux la languej'sais pas moi/l'arabe littéraire parfois ça dépend la situation/ça dépend la situation/moi ce quim'intéresse/je sais pas moi/c'est transmettre le message à l'élève faire sortit ou sauver l'élève de cette situation du blocage (E.4)

-Quand il s'agit d'un blocage ça y est/j'essaie plusieurs moyens mais rien ou il n'a pas compris/ il n'a pas pu puis j'ai le mot je sais pas moi je débloque je débrouille (rire) (E.4)

-Le dialecte nous sauvel 
L'enseignant $\mathrm{n}^{\circ} \mathbf{5}$ développe une « conscience linguistique» dans la mesure où elle explique que le dialecte comprend un certain nombre de mots empruntés à la langue française, c'est à travers ces mots que le recours à LR semble être plus avantageux.

-ily a un dialecte où où où/le le le le mélange se trouve entre peut-être l'arabe et le français/là là le dialecte qui qui a une certaine source qui vient du français là c'est utile c'est utile sauf dans la prononciation qui n'est pas bien prononcél n'est pas bien formulé/là on on c't'à dire on en profite on en profite pour dire voilà comment le même mot/sauf à la maison dit autrement lécole ou bien y a beaucoup de mot/y a cosina/y a tabla/ y a beaucoup de mots/ bioche c'est la pioche/ bala c'est la pelle/ tous les moyens/si si vous considérez balle la pelle cosina bioche considérés comme dialecte/là c'est utile/ là c'est utile pa[R]c'que pa[R]c'que là on va pas passer beaucoup de temps pour expliquer ces mots/on va passer peu de temps pour l'orienter pour le régler pour mettre c't'à dire la bonne prononciation (E.5)

-Bien sûr/bien sûr/çajoue un rôle pédagogique/çajoue un rôle pédagogique (E.5)

-le dialecte peut l'utiliser comme moyen d'explication du contexte/ ou bien ou du du vocabulaire des mots (E.5)

Pour le même enquêté, le recours à l'arabe dialectal peut économiser le temps de l'explication et faciliter l'accès à la compréhension des mots, car il est vu comme stratégie «rapide» et efficace pour poursuivre son cours.

-Là c'est un moyen/ c'est un moyen pa[R]c'que on va pas/on va pas/on va pas perdre le temps à expliquer le mot/ la compréhension du mot est déjà acquise/ mais il reste l'écriture des mots/ l'écriture des mots/ ou bien la prononciation juste en français/là c'est pas difficile/comme facer effacer (E.5) -si je vais l'utiliser pour que je sorte de l'ambiguitél pour que je minimise le temps/ pa[R]c'que le mot étudié qu'on veut comprendre prendra beaucoup de temps/toute une situation pour l'expliquer/là peut-être là je vais le dire/ puisque l'accès au sens se ferait plus rapidement en dialectal que l'explication en langue française/ beaucoup de temps pa[R]c'que comme vous savez la compréhension du mot est très sensible très sensible/ $(\mathrm{E} .5)$ 


\subsection{Fonction ludique}

La présence de LR peut jouer un rôle de délassement, dans la mesure où elle suscite le désir d'apprendre et brise la monotonie surmenante de la classe.

-ça m'arrive de l'utiliser/ et ça va aider/ et bien sûr/ bien sûr c'est un moment de répit/ c'est un moment de loisir/ c'est un moment de loisir/ bien sûr que je vais le dire/ les enfants vont rire// c'est une pause (E.5)

-des fois/des fois je vais I'utiliser pour sortir du sérieux/c'est tout pour rire (E.5)

- (monsieur combien de giga ?) (rire) j'ai saisi l'occasion de rire/j'ai pas j'ai pas réagi (E.5)

-la langue à double tranchant besoin pédagogique et d'amusement (E.5)

-ça fait vraiment rire/ donc voyez c'est un moment de distraction !/c'est un mot de distraction/ de divertissement/(E.5)

-Peut-être que/ écoutez ! des fois des fois ça m'arrive ça m'arrive de l'utiliser/ et ça va aider et bien sûr/bien sûr bien sûr c'est un moment/c'est un moment de répit/ c'est un moment de loisir/ c'est un moment de loisir/ bien sûr que je vais le dire/ les enfants vont rire/ les élèves vont rire/ et c'est un moment de/ et c'est un moment d'une part/ d'une part c'est une pause pour que le// j'ai utilisé des fois ce dialecte pour j'ai dit/ pour d'une part c't'à dire d'une part/ c'est un moment de pause/ pour les élèves vont sortir des règles/vont sortir de de de de/ on peut pas dire la routine/ du sévère du sérieux donc ils vont rire (E.5)

\subsection{Fonction coercitive « insultante»}

L'usage de LR, en classe, se conçoit comme outil régulateur ayant pour objectif de gérer une situation, à titre illustratif l'enseignante emploie certains vocables de l'arabe dialectal pour avertir l'élève ou capter son attention au cours de la séance.

-j'utilise l'arabe/ mais l'arabe lorsque je veux insulter/ lorsque je veux (rire) فهنتي (vous m'avez compris) لخاطر) sije parle en français ils ne vont pas comprendre/نعايرهم بالاخر لازم (il faut les insulter) en arabe/ (E.2)

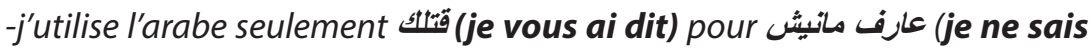
pas) pour crier (E.2) 


\subsection{Fonction socialisante et libératrice de la parole}

Le recours à LR peut accomplir une fonction de socialisation : lorsque l'enseignant parle l'arabe dialectal, il inscrit explicitement l'apprenant dans son contexte sociétal et le libère du «joug » accablant la parole.

-des fois/des fois je vais l'utiliser seulement pour sortir du sérieux/ c'est tout pour rire pour faire référence/ qui sommes-nous? Nous sommes des Algériens/ nous utilisons dans nos maisons ce dialecte/là là je je c't'à dire que que que/ je peux accepter mais je le confirme pas/je le confirme pas voilà/ c't'à dire ça reste toujours/de faire sortir l'apprenant des règles et des normes de la phrase/ de la classe/ pa[R]c'que l'élève a besoin de respirer un peu et puis le fait de sortir/ de sortir de de la langue/ du cadre et de rendre encore une fois/ c'est une mise au point/ c'est une mise au point/ c'est-à-dire l'élève va sortir et va revenir/est-ce qu'il n'a pas oublié ?/ est-ce qu'il maîtrise encore ?/ quelle est sa capacité ?/ quelle est sa capacité de de de revenir au sérieux ?/ là là c't'à dire que le professeur doit maîtriser ça (E.5)

-Il faut pour moi pour moi/ oui bien sûr pour lui c'est une occasion de se retrouver à la maison/ occasion de se libérer de cette langue qui l'accable/ cette langue qui lui attache les mains/ la bouche à travers ses règles donc pour lui/ il faut qu'il s'évade/ qu'il parle encore (E.5)

\subsection{Propriété sémantique intraduisible}

Les enquêtés $\mathrm{n}^{\circ} \mathbf{1}$ et $\mathrm{n}^{\circ} \mathbf{4}$ déclarent que le mot a un caractère polysémique dont le sens change selon le contexte d'usage et son origine culturo-linguistique, c'est pour quoi le recours à LR est déterminant. Il existe des mots qui sont intraduisibles ou, au moins, ont un sens si restreint.

-La phrase elle est toujours/ ou bien une phrase elle est toujours l'a plusieurs sens/d'accord pour moi c'est mon avis personnel un mot n'a pas de sens/le mot est polysémique/ le mot n'a pas de sens/la différence d'utilisation est change de signification selon I'utilisation (E.1)

-On doit chercher le mot dans l'arabe dialectal/ pas l'arabe littéraire ou académique (E.1)

-Pour bien exprimer ou bien transmettre ou bien/ il vaut mieux utiliser le dialecte (E.4) 


\begin{tabular}{|c|c|}
\hline $\mathbf{N}^{\circ} \mathbf{E}$. & Fréquence des vocables de la langue de référence dans les discours des enquêtés \\
\hline E. 1 & 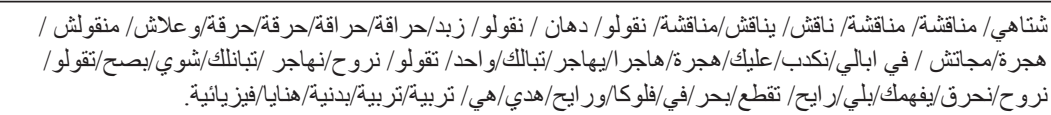 \\
\hline E. 2 & 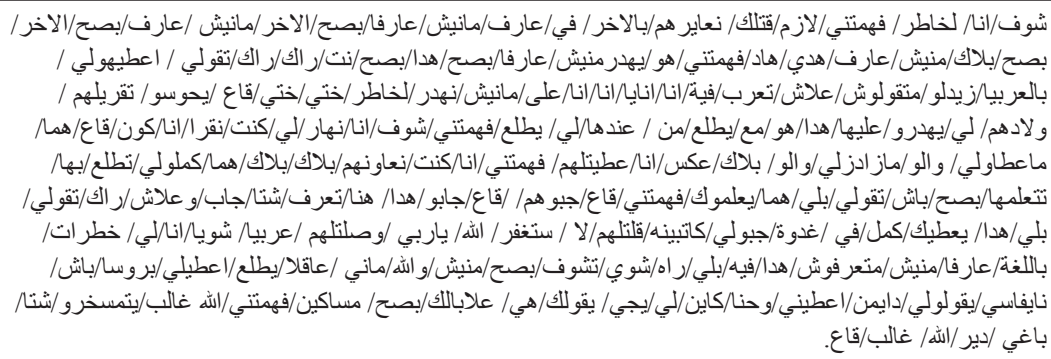 \\
\hline E. 3 & صح/ز عما/حرقا/سلام/سلام/ عليكم/حقبة/حقبة/صح/في/انا/و الش/و لا/و لا \\
\hline E. 4 & هجر //هر ا/حرقا/هي/حر قا/لي/كانت/كنا/نفهمو/عندها/حالا/الاستادة/علاش/تفهنا/كانت/نقولو /بز اف/هو/هذا \\
\hline E. 5 & حرقة/حرقا/حر قة/حرقا /نطلع/نفاسيها/اشيخ/شحال/من / جيقو ات /اشيخ/شحال/من/جيقوات/جيقوات /شحال /من/جيقوات / \\
\hline
\end{tabular}

\section{Tableau 2: Continuum linguistique des enseignants}

\begin{tabular}{|l|c|c|c|c|c|}
\hline Enseignants & E. 1 & E. 2 & E. 3 & E. 4 & E. 5 \\
\hline Nombre de vocables de LR & 51 & 177 & 14 & 19 & 21 \\
\hline
\end{tabular}

\section{Discussion et Conclusion}

A l'issue de cette analyse, nous avons pu constater que le nombre de vocables de LR varie considérablement d'un enseignant à l'autre. II se répartit effectivement de façon inégale allant d'un taux trop élevé, soit le nombre $\mathbf{1 7 7}$ mots chez E. 2 ; $\mathbf{5 7}$ chez E.1, 21 mots chez E. 5 ; 19 chez E. 4 jusqu'à moindre fréquence 14 chez E. 3. La présence de ces vocables dans les discours des enquêtés révèle la véritable identité des enseignants, ladite identité plurilingue.

Cela tend à confirmer notre hypothèse émise au départ, au-delà de l'usage fréquent de LR, les enseignants s'en font des représentations « négatives » en classe de FLE.

Il est à noter que la comparaison entre les déclarations des enseignants et leurs discours effectifs (la fréquence des vocables de LR) montre un « hiatus » ou une discrépance entre les représentations qu'ils se font de LR et la pratique langagière réelle, 
par exemple les enseignantes $\mathbf{2}$ et $\mathbf{3}$ déclarent qu'elles n'utilisent l'arabe que dans des situations-problèmes alors que leurs discours sont marqués par la présence des vocables de LR. Ainsi, l'enseignante $\mathbf{4}$ déclare qu'elle n'utilise nullement l'arabe dialectal, or son discours révèle un certain nombre de vocables arabes. Le même constat est dévoilé dans les discours des enseignants $\mathbf{1}$ et $\mathbf{5}$.

Du reste, nous avons pu montrer le «fossé» entre les «représentations négatives » que portent les enseignants sur LR et l'usage qu'ils en font en classe, à titre indicatif les enseignants $\mathbf{4}$ et $\mathbf{5}$ conçoivent que l'intégration de cette langue en classe est inutile tandis que leurs déclarations verbales montrent que certains vocables relèvent de LR.

Cela démontre que le monolinguisme n'est qu'une représentation, c'est-à-dire une idée que l'enseignant se fait de ce qui doit se dire dans la classe, alors que l'usage démontre un fait contraire (un plurilinguisme de fait).

Évaluation : Évaluation anonyme par des pairs extérieurs.

Conflit d'intérêts : Les auteurs n'ont aucun conflit d'intérêts à déclarer.

Subvention : Les auteurs n'ont reçu aucun soutien financier pour ce travail.

Peer-review: Externally peer-reviewed.

Conflict of Interest: The authors has no conflict of interest to declare.

Grant Support: The authors declared that this study has received no financial support.

\section{Bibliographie}

Blanchet, Ph. et Martinez, P. (2010). Pratiques innovantes du plurilinguisme : émergence et prise en compte en situation francophone. Paris: EAC.

Bourdieu, P. (1982). Ce que parler veut dire, L'économie des échanges linguistiques. Paris: Fayard.

Boyer, H. (2001). Introduction à la sociolinguistique. Paris: Dunod.

Castellotti, V. et Moore, D. (2002). Représentations sociales des langues et enseignements : Guide pour l'élaboration des politiques linguistiques éducatives en Europe - De la diversité linguistique à l'éducation plurilingue. Strasbourg: Conseil de l'Europe.

Elimam, A. (2015). Le maghribi, alias « ed-derija » (La langue consensuelle du Maghreb). Tizi-Ouzou: Éditions FrantzFanon.

Jodelet, D. (1989). Les représentations sociales. Paris: Presses Universitaires de France.

Rispail, M. (dir.). (2017). Abécédaire de sociodidactique. 65 notions et concepts. Publications de l'Université de Saint-Etienne.

Sebaa, R. (2015). L'Algérie et la langue française ou l'altérité en partage. Tizi-Ouzou: Frantz-Fanon. 
